\title{
Analytic response theory for the density matrix renormalization group
}

Jonathan J. Dorando, Johannes Hachmann, and Garnet Kin-Lic Chan

Citation: J. Chem. Phys. 130, 184111 (2009); doi: 10.1063/1.3121422

View online: http://dx.doi.org/10.1063/1.3121422

View Table of Contents: http://aip.scitation.org/toc/jcp/130/18

Published by the American Institute of Physics 


\title{
Analytic response theory for the density matrix renormalization group
}

\author{
Jonathan J. Dorando, Johannes Hachmann, and Garnet Kin-Lic Chan ${ }^{\text {a) }}$ \\ Department of Chemistry and Chemical Biology, Cornell University, Ithaca, New York 14853-1301, USA
}

(Received 20 January 2009; accepted 23 March 2009; published online 13 May 2009)

\begin{abstract}
We propose an analytic response theory for the density matrix renormalization group, whereby response properties correspond to analytic derivatives of density matrix renormalization group observables with respect to the applied perturbations. Both static and frequency-dependent response theories are formulated and implemented. We evaluate our pilot implementation by calculating static and frequency-dependent polarizabilities of short oligodiacetylenes. The analytic response theory is competitive with dynamical density matrix renormalization group methods and yields significantly improved accuracies when using a small number of density matrix renormalization group states. Strengths and weaknesses of the analytic approach are discussed. (C) 2009 American Institute of Physics. [DOI: 10.1063/1.3121422]
\end{abstract}

\section{INTRODUCTION}

The density matrix renormalization group (DMRG) $\operatorname{method}^{1}$ is now established as a powerful tool for "difficult" electronic structure problems in physics and chemistry. ${ }^{2-5}$ In molecular systems, it has been used to describe multireference correlation in medium-sized active spaces (20-30 active orbitals) for small molecules with complex bonding, ${ }^{6-9}$ as well as a local multireference correlation method in extended long-chain molecules, e.g., to describe excited states in conjugated molecules, using large active spaces of up to 100 active orbitals. $^{10}$

Response properties, which represent the change in an observable as a function of an applied perturbation, are of interest in many physical and chemical applications. For example, geometry optimization and vibrational frequencies both require the response of the energy with respect to changes in the nuclear coordinates, quantities usually known as nuclear derivatives. Nuclear derivatives are examples of static response properties because the perturbation does not depend on time. It is also common to consider frequencydependent (i.e., dynamical) response properties where the applied perturbation is a function of time. The most common time-dependent perturbations are fluctuating electric and/or magnetic fields. In extended systems, the frequency dependence of the response gives insight into the elementary excitations of the system and this can be used to characterize the nature of the electronic ground state. ${ }^{11}$

In many electronic structure methods, response properties are obtained by the so-called "analytic" techniques. Analytic response theories of this kind at linear and higher orders have been developed and implemented for most electronic structure methods including Hartree-Fock, ${ }^{12}$ density functional, ${ }^{13}$ coupled cluster $(\mathrm{CC}),{ }^{14}$ multiconfigurational self-consistent, ${ }^{15}$ and Møller-Plesset perturbation theories. ${ }^{16}$ A review of the formal theory and some of these developments may be found in Ref. 17. The name analytic is

\footnotetext{
${ }^{a)}$ Author to whom correspondence should be addressed. Electronic mail: gc238@cornell.edu.
}

used because the response properties evaluated (e.g., the perturbed energies) correspond strictly to derivatives of the ground-state energies or quasienergies ${ }^{17-19}$ evaluated in the presence of the perturbation using the same level of approximation for the (quasi) energy with and without the perturbation.

In contrast, response properties in the DMRG have typically been obtained using a quite different approach that appears natural within the DMRG. In the DMRG, the wave function is expanded in a set of many-electron states that are adapted to the state of interest. To obtain a response property, one can choose to solve response equations using basis states that are adapted not only to the zeroth-order state but also to the calculation of the state's response. These response methods, which have proven very useful in the calculation of dynamical response in DMRG model Hamiltonian calculations, go by the names of Lanczos-vector DMRG ${ }^{20}$ correction vector DMRG, ${ }^{21,22}$ and dynamical DMRG. ${ }^{23}$ More recently, explicit real-time propagation of the DMRG equations has also been used to obtain high-frequency response properties. ${ }^{24}$ A recent review of all these DMRG response methods can be found in Ref. 25.

In the current work we return to an analytic formulation of the response theory within the DMRG, in a way that parallels the description of response properties in other electronic structure methods. We use as our starting point the wave function based (matrix-product state) formulation of the DMRG. ${ }^{2,5,26,27}$ As we shall see, the analytic response approach has a number of strengths and weaknesses compared to earlier DMRG response methods. To understand these strengths and weaknesses better, we perform a series of benchmark static and frequency-dependent polarizability calculations on oligodiacetylenes (ODAs) that compare the behavior of the earlier dynamical DMRG method with our analytic response DMRG approach. Using our data, we examine the scaling of the polarizability as a function of the number of monomer units. 


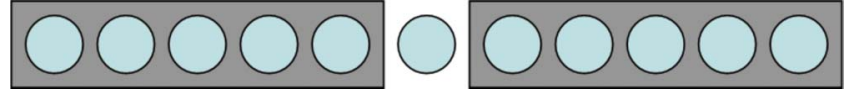

FIG. 1. (Color online) One-site DMRG block configuration. $\mathbf{L}^{n}$ tensors are associated with the left block, $\mathbf{R}^{n}$ tensors with the right block, and the middle site is site $p$ in Eq. (1).

\section{TIME-INDEPENDENT AND TIME-DEPENDENT DMRG EQUATIONS}

The DMRG works with a variational ansatz for the wave function $\Psi$. The simplest ansatz to analyze is the "one-site" form of the DMRG wave function. ${ }^{4,27,28}$ For the blockconfiguration depicted in Fig. 1, the wave function takes the form

$$
|\Psi\rangle=\sum_{\{n\}} \mathbf{L}^{n_{1}} \cdots \mathbf{L}^{n_{p-1}} \mathbf{C}^{n_{p}} \mathbf{R}^{n_{p+1}} \cdots \mathbf{R}^{n_{k}}\left|n_{1} \cdots n_{k}\right\rangle .
$$

The $\mathbf{L}^{n}$ and $\mathbf{R}^{n}$ renormalization tensors satisfy the orthogonality conditions

$$
\begin{aligned}
& \sum_{n} \mathbf{L}^{n \dagger} \mathbf{L}^{n}=\mathbf{1}, \\
& \sum_{n} \mathbf{R}^{n} \mathbf{R}^{n \dagger}=\mathbf{1},
\end{aligned}
$$

and formally define the sequence of renormalization transformations to obtain basis states $\{l\}$ and $\{r\}$ for the left and right blocks in Fig. 1. [Note that in Eqs. (2) and (3) we have dropped the subindices on $n$ as these conditions are not specific to any given site. We will use a similar convention throughout to avoid a proliferation of unnecessary indices.] The coefficient tensor $\mathbf{C}^{n}$ gives the expansion coefficients of the wave function in the superblock basis $\{l\} \otimes\left\{n_{p}\right\} \otimes\{r\}$. When viewed as a flattened vector $\mathbf{c}$, it satisfies the normalization condition $\mathbf{c}^{\dagger} \mathbf{c}=1$.

The DMRG energy is minimized when the tensors satisfy certain equations. For the coefficient vector, this is a time-independent effective Schrödinger equation

$$
\mathbf{H c}=E \mathbf{c},
$$

where the effective renormalized superblock Hamiltonian $\mathbf{H}$ satisfies $E=\langle\Psi|H| \Psi\rangle=\mathbf{c}^{\dagger} \mathbf{H c}$. The renormalization tensors at each position are defined from the coefficient tensor at the same position, i.e., $\mathbf{C}^{n}$ defines $\mathbf{L}^{n}$ and $\mathbf{R}^{n}$ via intermediate left and right density matrices. To obtain the left density matrix $\mathbf{D}_{L}$, we view the tensor $\mathbf{C}^{n}$ as a matrix $\mathbf{C}$ indexed by $(l n), r$, where $l$ is the row index of $\mathbf{C}^{n}$, then $\mathbf{D}_{L}=\mathbf{C C}^{\dagger}$. The right density matrix $\mathbf{D}_{R}$ is defined in a similar way, we view the tensor $\mathbf{C}^{n}$ as a matrix $\mathbf{C}$ indexed by $l,(n r)$, where $r$ is the column index of $\mathbf{C}^{n}$ and $\mathbf{D}_{R}=\mathbf{C}^{\dagger} \mathbf{C}$. The renormalization tensors $\mathbf{L}^{n}$ and $\mathbf{R}^{n}$, when viewed as matrices $\mathbf{L}$ and $\mathbf{R}$ in the appropriate way, are obtained from the $M$ eigenvectors (with largest weights) of the density matrices $\mathbf{D}_{L}$ and $\mathbf{D}_{R}$, respectively, i.e.,

$$
\mathbf{D}_{L} \mathbf{L}=\mathbf{L}\left(\sigma_{1} \cdots \sigma_{M}\right)_{\text {diag }}, \quad \sigma_{1} \geq \sigma_{2} \cdots \geq \sigma_{M}\left(\mathbf{L}_{(l n) i}=\mathbf{L}_{l i}^{n}\right),
$$

$\mathbf{D}_{R} \mathbf{R}=\mathbf{R}\left(\sigma_{1} \cdots \sigma_{M}\right)_{\text {diag }}, \quad \sigma_{1} \geq \sigma_{2} \cdots \geq \sigma_{M}\left(\mathbf{R}_{(r n) i}=\mathbf{R}_{i r}^{n}\right)$.

More explicitly, writing the eigenvectors of the left and right density matrices as $\mathbf{l}^{i}$ and $\mathbf{r}^{i}$,

$$
\begin{aligned}
& \mathbf{D}_{L} \mathbf{l}^{i}=\mathbf{l}^{i} \sigma_{i}, \quad \sigma_{1} \geq \sigma_{2} \geq \sigma_{3} \cdots, \\
& \mathbf{D}_{R} \mathbf{r}^{i}=\mathbf{r}^{i} \sigma_{i}, \quad \sigma_{1} \geq \sigma_{2} \geq \sigma_{3} \cdots .
\end{aligned}
$$

Here $\mathbf{L}^{n}$ and $\mathbf{R}^{n}$ are constructed by assigning the elements of the eigenvectors to the tensors in the following way:

$$
\begin{aligned}
\mathbf{L}_{j i}^{n} & =\mathbf{l}_{(n j)}^{i}, \quad i=1, \ldots, M, \\
\mathbf{R}_{i j}^{n} & =\mathbf{r}_{(n j)}^{i}, \quad i=1, \ldots, M .
\end{aligned}
$$

In Ref. 29, we showed that satisfying the solution conditions [Eqs. (4)-(6)] for $\mathbf{C}^{n}, \mathbf{L}^{n}$, and $\mathbf{R}^{n}$ is formally equivalent to minimizing the DMRG energy subject to normalization and the orthogonality constraints [Eqs. (2) and (3)]. We can formally extend the DMRG theory to time-dependent scenarios by making stationary the Dirac-Frenkel action $\langle\Psi|i \partial / \partial t-H| \Psi\rangle$ (Ref. 12) subject to the same normalization and orthogonality constraints. (Interestingly, the DiracFrenkel action has recently been independently rederived in the DMRG context in Ref. 30). For the coefficient vector, the time evolution is then given by an effective time-dependent Schrödinger equation

$$
i \partial_{t} \mathbf{c}=\mathbf{H} \mathbf{c} .
$$

The corresponding $\mathbf{L}^{n}$ and $\mathbf{R}^{n}$ remain defined by Eqs. (5) and (6).

\section{COUPLED-PERTURBED DMRG RESPONSE EQUATIONS}

We now consider the effect of an external perturbation. We start with a time-independent perturbation $V$. In the superblock basis $\{l\} \otimes\left\{n_{p}\right\} \otimes\{r\}$, this yields the effective perturbation $\mathbf{V}$, which satisfies $\langle\Psi|V| \Psi\rangle=\mathbf{c}^{\dagger} \mathbf{V} \mathbf{c}$.

In response to this perturbation, the $\mathbf{L}^{n}, \mathbf{C}^{n}$, and $\mathbf{R}^{n}$ tensors each can be expanded in orders of $|V|$, giving

$$
\begin{aligned}
& \mathbf{L}^{n}=\mathbf{L}^{n[0]}+\mathbf{L}^{n[1]}+\cdots, \\
& \mathbf{C}^{n}=\mathbf{C}^{n[0]}+\mathbf{C}^{n[1]}+\cdots, \\
& \mathbf{R}^{n}=\mathbf{R}^{n[0]}+\mathbf{R}^{n[1]}+\cdots,
\end{aligned}
$$

Thus the first-order DMRG wave function for the block configuration in Fig. 1 takes the general form

$$
\begin{aligned}
\left|\Psi^{[1]}\right\rangle= & \sum_{\{n\}}\left[\left(\mathbf{L}^{n_{1}[1]} \cdots \mathbf{C}^{n_{p}[0]} \cdots \mathbf{R}^{n_{k}[0]}\right)+\cdots\right. \\
& +\left(\mathbf{L}^{n_{1}[0]} \cdots \mathbf{C}^{n_{p}[1]} \cdots \mathbf{R}^{n_{k}[0]}\right)+\cdots \\
& \left.+\left(\mathbf{L}^{n_{1}[0]} \cdots \mathbf{C}^{n_{p}[0]} \cdots \mathbf{R}^{n_{k}[1]}\right)\right]\left|n_{1} n_{2} \cdots n_{k}\right\rangle .
\end{aligned}
$$

We now derive the response equations satisfied by each of the quantities $\mathbf{L}^{n[1]}, \mathbf{C}^{n[1]}$, and $\mathbf{R}^{n[1]}$. These are obtained by the perturbation expansion of the solution conditions (4)-(6). For the coefficient vector, this yields 


$$
\begin{gathered}
\left(\mathbf{H}^{[0]}+\Delta \mathbf{H}^{[1]}+\mathbf{V}^{[1]}+\cdots\right)\left(\mathbf{c}^{[0]}+\mathbf{c}^{[1]}+\cdots\right) \\
=\left(E^{[0]}+E^{[1]}+\cdots\right)\left(\mathbf{c}^{[0]}+\mathbf{c}^{[1]}+\cdots\right) .
\end{gathered}
$$

Note the first-order change in the Hamiltonian $\Delta \mathbf{H}^{[1]}$. This arises because the effective Hamiltonian in the superblock basis $\mathbf{H}$ depends on the renormalization tensors $\mathbf{L}^{n}$ and $\mathbf{R}^{n}$ (which define the renormalized basis) and so first-order changes in those tensors lead to a first-order change in the effective Hamiltonian. (The construction of $\Delta \mathbf{H}^{[1]}$ is described later in Sec. IV). Gathering first-order terms and enforcing intermediate normalization through the projector $\mathbf{Q}=\mathbf{1}-\mathbf{c}^{[0]} \mathbf{c}^{[0] \dagger}$ gives

$$
\left(\mathbf{H}^{[0]}-E^{[0]} \mathbf{1}\right) \mathbf{c}^{[1]}=-\mathbf{Q}\left(\boldsymbol{\Delta} \mathbf{H}^{[1]}+\mathbf{V}^{[1]}\right) \mathbf{c}^{[0]} .
$$

Because $\boldsymbol{\Delta} \mathbf{H}^{[1]}$ depends on the first-order wave function through its dependence on the $\mathbf{L}^{n}$ and $\mathbf{R}^{n}$ tensors, Eq. (17) must be solved self-consistently. It is therefore a coupledperturbed response equation, analogous to the coupledperturbed orbital equations that arise in the Hartree-Fock theory of response.

The first-order coefficients $\mathbf{C}^{n[1]}$ define the first-order renormalization tensors at the same site $\mathbf{L}^{n[1]}$ and $\mathbf{R}^{n[1]}$. Viewing $\mathbf{C}^{n[0]}$ and $\mathbf{C}^{n[1]}$ as matrices in the appropriate fashion, we obtain first-order left and right density matrices

$$
\begin{array}{ll}
\mathbf{D}_{L}^{[1]}=\mathbf{C}^{[0]} \mathbf{C}^{[1] \dagger}+\mathbf{C}^{[1]} \mathbf{C}^{[0] \dagger}, & \left(\mathbf{C}_{(n l), r}=\mathbf{C}_{l r}^{n}\right), \\
\mathbf{D}_{R}^{[1]}=\mathbf{C}^{[0]^{\dagger}} \mathbf{C}^{[1]}+\mathbf{C}^{[1] \dagger} \mathbf{C}^{[0]}, & \left(\mathbf{C}_{l,(n r)}=\mathbf{C}_{l r}^{n}\right) .
\end{array}
$$

In response to the change in the density matrices, the eigenvectors have a perturbation expansion

$$
\begin{aligned}
& \mathbf{l}^{i}=\mathbf{l}^{i[0]}+\mathbf{l}^{i[1]}+\cdots, \\
& \mathbf{r}^{i}=\mathbf{r}^{i[0]}+\mathbf{r}^{i[1]}+\cdots,
\end{aligned}
$$

and we can set up corresponding response equations

$$
\begin{aligned}
& \left(\mathbf{D}_{L}^{[0]}-\sigma_{i} \mathbf{1}\right) \mathbf{l}^{i[1]}=-\mathbf{Q}_{L} \mathbf{D}_{L}^{[1]} \mathbf{l}^{i[0]}, \\
& \left(\mathbf{D}_{R}^{[0]}-\sigma_{i} \mathbf{1}\right) \mathbf{r}^{i[1]}=-\mathbf{Q}_{R} \mathbf{D}_{R}^{[1]} \mathbf{r}^{i[0]},
\end{aligned}
$$

where $\mathbf{Q}_{L}$ and $\mathbf{Q}_{R}$ project out the span of $\mathbf{D}_{L}$ and $\mathbf{D}_{R}$, respec-

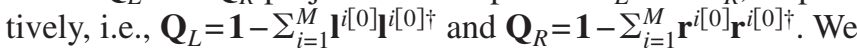
assign the elements of each of the $M$ perturbed vectors $\mathbf{I}^{i[1]}$ and $\mathbf{r}^{i[1]}$ according to Eqs. (9) and (10) to define $\mathbf{L}^{n[1]}$ and $\mathbf{R}^{n[1]}$. The response equations for a time-dependent perturbation may be obtained in an analogous way as above. We consider for simplicity a perturbation with a single Fourier component,

$$
V(t)=V e^{i \omega t}+V^{*} e^{-i \omega t} .
$$

We expand the $\mathbf{L}^{n}, \mathbf{C}^{n}$, and $\mathbf{R}^{n}$ tensors in terms of the orders of $|V|$,

$$
\begin{aligned}
& \mathbf{L}^{n}(t)=\left(\mathbf{L}^{n[0]}+\mathbf{L}^{n[1]}(t)+\cdots\right) e^{-i E^{[0]} t}, \\
& \mathbf{C}^{n}(t)=\left(\mathbf{C}^{n[0]}+\mathbf{C}^{n[1]}(t)+\cdots\right) e^{-i E^{[0]_{t}},}
\end{aligned}
$$

$$
\mathbf{R}^{n}(t)=\left(\mathbf{R}^{n[0]}+\mathbf{R}^{n[1]}(t)+\cdots\right) e^{-i E^{[0]} t} .
$$

For the coefficient vector, we substitute this expansion into the effective time-dependent Schrödinger equation (11) and identify terms with frequencies $\omega$ and $-\omega$, giving

$$
\begin{aligned}
& \left(\mathbf{H}^{[0]}-\left(E^{[0]}+\omega\right) \mathbf{1}\right) \mathbf{c}^{[1]}(\omega)=-\mathbf{Q}\left(\Delta \mathbf{H}^{[1]}(\omega)+\mathbf{V}^{[1]}\right) \mathbf{c}^{[0]}, \\
& \left(\mathbf{H}^{[0]}-\left(E^{[0]}-\omega\right) \mathbf{1}\right) \mathbf{c}^{[1]}(-\omega) \\
& \quad=-\mathbf{Q}\left(\Delta \mathbf{H}^{[1]}(-\omega)+\mathbf{V}^{[1] *}\right) \mathbf{c}^{[0]},
\end{aligned}
$$

where $\mathbf{Q}$ is the projector defined in Eq. (17). The first-order frequency perturbed wave functions then define first-order perturbed density matrices $\mathbf{D}_{L}(\omega), \mathbf{D}_{L}(-\omega), \mathbf{D}_{R}(\omega)$, and $\mathbf{D}_{R}(-\omega)$ which can be used to obtain $\mathbf{L}^{n[1]}(\omega), \mathbf{L}^{n[1]}(-\omega)$, $\mathbf{R}^{n[1]}(\omega)$, and $\mathbf{R}^{n[1]}(-\omega)$ through Eqs. (22) and (23).

\section{A. Response properties}

Once we obtain the first-order response of the DMRG wave function, we can evaluate response properties of interest. We take as our example here the dipole-dipole response function or polarizability. For a uniform static electric field $\mathcal{E}_{i}$, the dipole moment is expanded as

$$
\mu_{i}=\mu_{i}^{[0]}+\sum_{j} \alpha_{i j} \mathcal{E}_{j}+\cdots, \quad i, j \cdots \in x, y, z,
$$

which defines the static polarizability $\alpha_{i j}$ as the first-order change in the dipole moment. Within the DMRG response theory, the polarizability is therefore obtained as

$$
\alpha_{i j}=\mathbf{c}^{[0] \dagger} \mu_{i}^{[0]} \mathbf{c}_{j}^{[1]}+\mathbf{c}_{j}^{[1] \dagger} \mu_{i}^{[0]} \mathbf{c}^{[0]}+\mathbf{c}^{[0] \dagger} \mu_{i(j)}^{[1]} \mathbf{c}^{[0]} .
$$

Here $\mu_{i}$ is the effective dipole operator in the superblock basis and $\mathbf{c}_{j}^{[1]}$ is the first-order wave function in response to an electric field in the $j$ direction. Note the additional contribution $\mu_{i(j)}^{[1]}$. This is the change in the effective dipole operator $\mu_{i}$ due to the response of the $\mathbf{L}^{n}$ and $\mathbf{R}^{n}$ tensors to an applied field in the $j$ direction. This quantity is constructed in a way similar to the effective Hamiltonian $\boldsymbol{\Delta} \mathbf{H}^{[1]}$.

For a frequency-dependent electric field $\mathcal{E}_{i}(t)$, we expand the dipole moment as

$$
\begin{aligned}
& \mu_{i}(t)=\mu_{i}^{[0]}+\sum_{j} \int d \omega e^{-i \omega t} \alpha_{i j}(\omega) \mathcal{E}_{j}(\omega)+\cdots, \\
& i, j \cdots \in x, y, z,
\end{aligned}
$$

where $\alpha_{i j}(\omega)$ and $\mathcal{E}_{j}(\omega)$ are the $\omega$ frequency components of the frequency-dependent polarizability and electric field. $\alpha_{i j}(\omega)$ contains two contributions: one from the $e^{i \omega t}$ component of the applied perturbation and one from the $e^{-i \omega t}$ component. The final expression for $\alpha_{i j}(\omega)$ therefore reads as

$\alpha_{i j}(\omega)=G_{i j}(\omega)+G_{i j}(-\omega)$, 
$G_{i j}(\omega)=\mathbf{c}^{[0] \dagger} \mu_{i}^{[0]} \mathbf{c}_{j}^{[1]}(\omega)+\mathbf{c}_{j}^{[1] \dagger}(\omega) \mu_{i}^{[0]} \mathbf{c}^{[0]}+\mathbf{c}^{[0] \dagger} \mu_{i(j)}^{[1]}(\omega) \mathbf{c}^{[0]}$.

Here $G_{i j}(\omega)$ and $G_{i j}(-\omega)$ are obtained from two separate response calculations, solving Eqs. (28) and (29), respectively.

\section{B. Comparison to other DMRG response theories}

So far we have derived a DMRG theory of response that was based on expanding the solution conditions satisfied by the DMRG wave function in terms of the applied perturbation. This corresponds to an analytic theory of response in the following way. Consider a time-independent perturbation for simplicity. Let us consider minimizing the energy of the DMRG wave function, for some fixed number of states $M$, with respect to the full Hamiltonian (with the perturbation) $H=H^{[0]}+\lambda V^{[1]}$, where $\lambda$ is used to scale the strength of the perturbation. This gives a wave function $\Psi(\lambda)$ and an energy $E(\lambda)$. The first-order wave function $\Psi^{[1]}$ and corresponding first-, second-, and third-order energies calculated with the analytic DMRG response theory correspond exactly to the following derivatives:

$$
\begin{aligned}
& \Psi^{[1]}=\left.\frac{\partial \Psi(\lambda)}{\partial \lambda}\right|_{\lambda=0}, \\
& E^{[1]}=\left.\frac{\partial E(\lambda)}{\partial \lambda}\right|_{\lambda=0}, \\
& E^{[2]}=\left.\frac{\partial^{2} E(\lambda)}{\partial \lambda^{2}}\right|_{\lambda=0}, \\
& E^{[3]}=\left.\frac{\partial^{3} E(\lambda)}{\partial \lambda^{3}}\right|_{\lambda=0} .
\end{aligned}
$$

Analogous statements for time-dependent perturbations can be made by considering an appropriate quasienergy. ${ }^{17-19}$

The analytic approach to DMRG response does not represent the only way to obtain response within the DMRG. Existing DMRG response methods use various related adaptive basis approaches, commonly in two categories, the Lanczos vector method ${ }^{20}$ and the dynamical DMRG. ${ }^{23}$ The dynamical DMRG is established as the most accurate approach to response properties and we shall focus on it here. [Note that the dynamical DMRG and correction vector methods $^{21,22,25}$ are essentially the same but differ in the algorithm used to solve the response equations. In fact, if the response quantities are evaluated using a quadratic functional of the correction vector such as Eq. (48), it is possible to obtain quadratic errors with the correction vector method without the explicit minimization as used in the dynamical DMRG.]

In the dynamical DMRG, the ansatz for the zeroth- and first-order wave functions is both modified relative to the unperturbed DMRG wave function, i.e.,

$$
\left|\Psi^{[0]}\right\rangle=\sum_{\{n\}} \widetilde{\mathbf{L}}^{n_{1}[0]} \cdots \widetilde{\mathbf{C}}^{n_{p}[0] \cdots} \widetilde{\mathbf{R}}^{n_{k}[0]}\left|n_{1} n_{2} \cdots n_{k}\right\rangle,
$$

$$
\left|\Psi^{[1]}\right\rangle=\sum_{\{n\}} \widetilde{\mathbf{L}}^{n_{1}[0]} \cdots \widetilde{\mathbf{C}}^{n_{p}[1] \cdots} \widetilde{\mathbf{R}}^{n_{k}[0]}\left|n_{1} n_{2} \cdots n_{k}\right\rangle
$$

The tildes indicate that the $\widetilde{\mathbf{L}}^{n}, \widetilde{\mathbf{C}}^{n}$, and $\widetilde{\mathbf{R}}^{n}$ tensors appearing in Eq. (39) even for the zeroth-order wave function do not correspond to the same tensors obtained in a DMRG calculation without the perturbation. The zeroth- and first-order coefficient vectors are obtained from the effective Schrödinger equation (4) and an uncoupled response equation, e.g.,

$$
\left(\mathbf{H}^{[0]}-\left(E^{[0]}+\omega\right) \mathbf{1}\right) \mathbf{c}^{[1]}(\omega)=-\mathbf{Q} \mathbf{V}^{[1]} \mathbf{c}^{[0]} .
$$

The dynamical DMRG ansatz is able to capture the response of the $\mathbf{L}^{n}$ and $\mathbf{R}^{n}$ tensors in an average way because it uses $\widetilde{\mathbf{L}}^{n}$ and $\widetilde{\mathbf{R}}^{n}$ that are different from those in the unperturbed DMRG calculation. Specifically, the left and right renormalization tensors at each block configuration are obtained as eigenvectors of modified left and right density matrices, where the density matrices corresponding to $\mathbf{c}^{[0]}, \mathbf{c}^{[1]}$, $\mathbf{v}=\mathbf{V}^{[1]} \mathbf{c}^{[0]}$ are all averaged together, i.e., for $\mathbf{D}_{L}$,

$$
\mathbf{D}_{L}=\alpha \mathbf{C}^{[0]} \mathbf{C}^{[0] \dagger}+\beta \mathbf{C}^{[1]} \mathbf{C}^{[1] \dagger}+\gamma\left(\mathbf{V} \mathbf{c}^{[0]}\right)\left(\mathbf{V} \mathbf{c}^{[0]}\right)^{\dagger},
$$

where $\alpha+\beta+\gamma=1$ and in the last term we are interpreting the perturbation multiplied by the zeroth-order wave function $\mathbf{V}^{[1]} \mathbf{c}^{[0]}$ as a matrix in the same way as $\mathbf{c}^{[0]}$ is interpreted as a matrix. (Note that the above is for real frequencies; when considering complex frequencies, one typically separates the imaginary and real contributions of the response vector. ${ }^{23}$ ) Because the density matrix contains information on the perturbation and the response, the DMRG basis is "adapted" to the perturbation being considered. While this is very simple to implement within a standard DMRG algorithm and has proven very successful, one drawback relative to the analytic response approach is that a single set of DMRG basis states is being used to represent several quantities, including both the zeroth-order and response vectors. For this reason, we can expect some loss of accuracy with this method for small $M$ calculations relative to the analytic response method.

\section{IMPLEMENTATION}

We have implemented the analytic DMRG response theory as described above. This consists of three parts: solving the coupled-perturbed equation (17) for the first-order coefficient vector $\mathbf{c}^{[1]}$, solving for the first-order renormalization tensors $\mathbf{L}^{n[1]}$ and $\mathbf{R}^{n[1]}$ [Eqs. (5) and (6)], and constructing the first-order effective Hamiltonian $\Delta \mathbf{H}^{[1]}$ and necessary intermediates, as well as other first-order operators needed for properties [e.g., $\mu_{i(j)}^{[1]}$ in Eq. (31)]. The first two parts are quite straightforward: we solve the coupled-perturbed equation (17) using a Krylov subspace iterative solver with preconditioning, and to obtain the first-order renormalization tensors (22) and (23), we use explicit Rayleigh-Schrödinger expressions for the first-order density matrix eigenvectors

$$
\mathbf{l}^{i[1]}=\sum_{j=M+1}-\frac{\mathbf{l}^{\mathbf{j}[0]^{\dagger} \mathbf{D}_{L}^{[1]} \mathbf{l}^{\mathbf{i}[0]}}}{\sigma_{j}^{[0]}-\sigma_{i}^{[0]}} \mathbf{j}^{[0]},
$$




$$
\mathbf{r}^{i[1]}=\sum_{j=M+1}-\frac{\mathbf{r}^{j[0]^{\dagger}} \mathbf{D}_{R}^{[1]} \mathbf{r}^{i[0]}}{\sigma_{j}^{[0]}-\sigma_{i}^{[0]}} \mathbf{r}^{j[0]} .
$$

In practice small denominators can arise in the perturbation expression (44); for stability we set contributions from any denominators below a certain threshold (e.g., $10^{-12}$ ) to zero.

We now focus on the implementation to obtain $\Delta \mathbf{H}^{[1]}$ and related quantities such as $\mu_{i(j)}^{[1]}$. We recall that the effective Hamiltonian $\mathbf{H}^{[0]}$ is expressed as a tensor product of operators on the left and right blocks (we consider the single site - in the block configuration Fig. 1 to be part of the left block for simplicity)

$$
\mathbf{H}=\sum_{i j} w_{i j} \mathbf{O}_{L}^{i} \otimes \mathbf{O}_{R}^{j},
$$

where $\mathbf{O}_{L}$ acts only on the left block and $\mathbf{O}_{R}$ acts only on the right block, and we assume that $\otimes$ takes into account the appropriate parity factors associated with the fermion character of the operators (see, e.g., Refs. 2 and 4). The firstorder Hamiltonian is constructed from the response of the operators $\mathbf{O}_{L}$ and $\mathbf{O}_{R}$, through

$$
\boldsymbol{\Delta} \mathbf{H}^{[1]}=\sum_{i j} w_{i j}\left(\mathbf{O}_{L}^{i[0]} \mathbf{O}_{R}^{i[1]}+\mathbf{O}_{L}^{i[1]} \mathbf{O}_{R}^{j[0]}\right) .
$$

We therefore need to calculate the first-order operators $\mathbf{O}_{L}^{[1]}$ and $\mathbf{O}_{R}^{[1]}$. These are built up sequentially through the blocking steps in the sweep much like the zeroth-order operators. The renormalization transformation $\mathcal{R}$ of the first-order operator at a given block configuration in a left $\rightarrow$ right sweep is given by

$$
\mathcal{R}\left[\underline{\mathbf{Q}}_{L}^{[1]}\right]=\mathbf{L}^{[0]} \underline{\mathbf{Q}}_{L}^{[1]} \mathbf{L}^{[0]}+\mathbf{L}^{[1] \dagger} \underline{\mathbf{Q}}_{L}^{[0]} \mathbf{L}^{[0]}+\mathbf{L}^{[0]} \underline{\mathbf{Q}}_{L}^{[0]} \mathbf{L}^{[1]},
$$

where we have used the underline to indicate that the operators refer to blocked operators (i.e., for the left block plus the single site), and the renormalization tensors are interpreted as matrices $\mathbf{L}$ as described in Eq. (5). At the beginning of the left $\rightarrow$ right sweep, $\mathbf{O}_{L}^{[1]}=0$ for all such operators. Analogous expressions hold for the right $\rightarrow$ left sweep and the operators $\mathbf{O}_{R}$.

The full sweep algorithm for the DMRG analytic response can be summarized as follows.

(1) Converge a standard DMRG algorithm for the state of interest and store all intermediate zeroth-order operators $\mathbf{O}_{L}^{[0]}$ and $\mathbf{O}_{R}^{[0]}$ and tensors $\mathbf{L}^{n[0]}, \mathbf{C}^{n[0]}$, and $\mathbf{R}^{n[0]}$.

(2) Set all $\mathbf{O}_{L}^{[1]}, \mathbf{O}_{R}^{[1]}=0$.

(3) Start a sweep (left $\rightarrow$ right).

- Set all $\mathbf{O}_{L}^{[1]}$ to 0 .

- At each block configuration:

- Solve coupled-perturbed response equation [Eq. (17)]. $\Delta \mathbf{H}^{[1]}$ is constructed using current best guesses for $\mathbf{O}_{L}^{[1]}$ and $\mathbf{O}_{R}^{[1]}$.

- Solve for perturbed density matrix eigenvectors and $\mathbf{L}^{n[1]}$ [Eq. (22)].

- Update all $\mathbf{O}_{L}^{[1]}$ using Eq. (47).
(4) Start a sweep (right $\rightarrow$ left), analogous to (left $\rightarrow$ right) sweep

(5) Loop to 3 until convergence.

(6) Evaluate response properties (e.g., as in Sec. III A).

We note that the cost of a single sweep for the analytic response has the same order of computational and storage cost as an ordinary sweep in the DMRG calculation, which, for the ab initio Hamiltonian is $O\left(M^{3} k^{3}\right)+O\left(M^{2} k^{4}\right)$ computation, $O\left(M^{2} k^{2}\right)$ memory, and $O\left(M^{2} k^{3}\right)$ disk, where $k$ is the number of correlated orbitals. The memory cost is roughly twice that for the calculation of the energy because of storage of the first-order operators as well as the zeroth-order operators.

We have assumed in the above that the coupledperturbed equations of the analytic DMRG response theory, i.e., Eqs. (17) and (29) can be solved through a simple selfconsistency cycle. In practice, however, we should expect convergence problems to occur when the first-order wave function $\mathbf{c}^{[1]}$ is large compared to $\mathbf{c}^{[0]}$, as this will lead to a large first-order effective Hamiltonian $\mathbf{\Delta} \mathbf{H}^{[1]}$ and a feedback effect in the response equations (17) and (29). This scenario arises near the poles of the response, and indeed we find this to be the case (see below).

\section{STATIC AND FREQUENCY-DEPENDENT POLARIZABILITIES OF ODAS}

As an initial test of the analytic DMRG response theory and implementation, we have calculated static and frequency-dependent longitudinal polarizabilities of several ODAs using the analytic DMRG response theory, the dynamical DMRG method, and the linear-response CC method. Long ODAs are of interest due to their large third-order nonlinear polarisability. ${ }^{31}$ While we will calculate only the linear polarizability here, the same analytic derivative techniques can, in principle, be extended to higher-order polarizabilities and nonlinear optical response.

We carried out calculations on short all-trans ODAs, 2-ODA $\mathrm{C}_{8} \mathrm{H}_{6}$, 4-ODA $\mathrm{C}_{16} \mathrm{H}_{10}$, and 6-ODA $\mathrm{C}_{24} \mathrm{H}_{14}$. Optimized geometries were obtained at the density functional theory B3LYP ${ }^{32,33}$ level in a correlation consistent Dunning double-zeta (cc-pVDZ) basis. ${ }^{34}$ Subsequent Hartree-Fock, DMRG, and CC calculations were carried out in a minimal STO-6G Gaussian basis. ${ }^{34,35}$ We realize that this basis is too small for the quantitative calculation of polarizabilities, but it has been chosen to enable a preliminary study. Also, we note that qualitative trends in polarizabilities can be captured using rather small basis sets of split-valence quality. ${ }^{31}$ The Hartree-Fock calculations were used to determine molecular orbitals with $\sigma$ and $\pi$ character. All $\sigma$ orbitals were kept frozen in the DMRG response calculations, and the $\pi$ orbitals were localized. Calculated polarizabilities refer to the $\alpha_{x x}$ component, where the $x$-axis is aligned with the long moment of inertia axis of the molecules (see Fig. 2). The DMRG response calculations used an active space of $p_{z}$ orbitals only, corresponding to an (8e, 8orb) active space for 2-ODA, a (16e, 16orb) active space for 4-ODA, and a (24e, 24orb) active space for 6-ODA. For the analytic response DMRG calculations using $M$ states, we first converged a ground-state DMRG calculation with $M$ states using the one- 


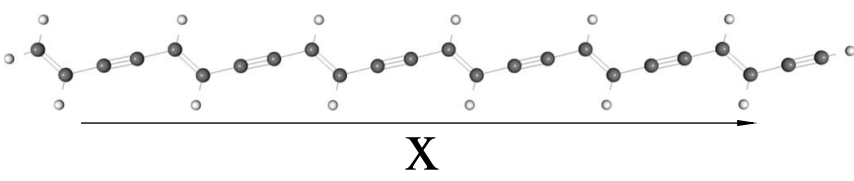

FIG. 2. ODAs with the long-axis moment of inertia aligned with the $x$-coordinate. This is the axis along which the polarizabilities are evaluated.

site algorithm and used this as the starting point for the response calculation.

In addition to the analytic response DMRG calculations, we carried out calculations using the dynamical DMRG method for comparison. The dynamical DMRG polarizabilities were obtained by solving the linear response equation in the dynamical DMRG basis $\left.\left(\omega \mathbf{1}-\mathbf{H}^{[0]}\right) \mathbf{c}^{[1]}\right)_{i}=\mathbf{Q} \mu_{i} \mathbf{c}^{[0]}$ just as in the correction vector method, but the resulting polarizabilities were evaluated using the quadratic functional

$$
G_{i j}=\mathbf{c}_{i}^{[1] \dagger}\left(\omega \mathbf{1}-\mathbf{H}^{[0]}\right) \mathbf{c}_{j}^{[1]}+\mathbf{c}^{[0] \dagger} \mu_{i} \mathbf{c}_{j}^{[1]}+\mathbf{c}_{j}^{[1] \dagger} \mu_{i} \mathbf{c}^{[0]} j,
$$

which ensures that the obtained polarizability is quadratic in the error in $\mathbf{c}^{[1]},{ }^{16,37}$ which is the hallmark of the dynamical DMRG approach. For comparison, we also computed linearresponse restricted $\mathrm{CC}$ polarizabilities at the singles and doubles level, ${ }^{14}$ both at the all electron level, and within the $p_{z}$ active space only, using the PSI3 (Ref. 38) package.

We note one issue that arises with the response DMRG calculations in our initial implementation as opposed to or- dinary ground-state DMRG calculations. In ground-state DMRG calculations with the one-site algorithm, we are generally able to converge the DMRG energy from sweep to sweep to very high accuracy, e.g., nanohartrees. However, in our initial response implementation, we were not able to converge the calculated polarizabilities to similar accuracy. Typically the forward and backward sweeps would converge to somewhat different results and even between consecutive forward (or backward) sweeps, the polarizability would oscillate somewhat. This was true both for the dynamical DMRG and the analytic response DMRG calculations. The oscillation can be quite severe, particularly for small $M$ calculations and for higher frequencies that are nearer to a pole (e.g., at frequency $\omega=0.2$ a.u.) and reflects the greater sensitivity of the response calculation to the discarded states in the density matrix. In our results, we report the average polarizability of the last four sweeps, together with twice the standard deviation. These results are reported in Table I.

From Table I we make the following observations about the relative performance of the analytic DMRG response method relative to the dynamical DMRG method that has been commonly used. For small $M$ (e.g., $M=25$ ), the analytic DMRG response method is clearly superior. While the dynamical DMRG method produces poor polarizabilities for $M=25$ in error by more than $50 \%$ in some cases, the analytic DMRG polarizabilities are quite reasonable at $M=25$ and

TABLE I. Static and frequency-dependent polarizabilities in a.u. of ODAs, with 2, 4, and 6 monomers (2-ODA, 4-ODA, and 6-ODA). $D$ stands for dynamical DMRG, $A$ stands for analytic response theory. $\omega$ is the frequency (in a.u.), $M$ refers to the number of states in the DMRG wave function. The numbers in parentheses do not represent intrinsic truncation error from finite $M$ but represent the numerical convergence of the DMRG sweep since the forward and backward sweeps typically converge to slightly different results. The bracketed number is twice the standard deviation $(2 \sigma)$ in the last four forward and backward sweeps. See text for further discussion.

\begin{tabular}{|c|c|c|c|c|c|c|c|}
\hline \multirow[b]{2}{*}{$\omega$} & \multirow[b]{2}{*}{$M$} & \multicolumn{2}{|c|}{ 2-ODA } & \multicolumn{2}{|c|}{ 4-ODA } & \multicolumn{2}{|c|}{ 6-ODA } \\
\hline & & $D$ & $A$ & $D(2 \sigma)$ & $A(2 \sigma)$ & $D(2 \sigma)$ & $A(2 \sigma)$ \\
\hline \multirow[t]{5}{*}{0.00} & 25 & 52.77 & 52.89 & $144.16(0.03)$ & $145.21(0.04)$ & $354.28(17.96)$ & $243.65(0.06)$ \\
\hline & 50 & 52.89 & 52.89 & $146.07(0.01)$ & $145.74(0.09)$ & $246.04(0.02)$ & $245.06(0.07)$ \\
\hline & 250 & 52.88 & 52.88 & $145.75(0.01)$ & $145.80(0.01)$ & $245.20(0.00)$ & $245.27(0.03)$ \\
\hline & 1000 & NA & NA & $145.77(0.01)$ & $145.81(0.00)$ & $245.13(0.10)$ & $245.14(0.02)$ \\
\hline & LR-CCSD & \multicolumn{2}{|c|}{53.38} & \multicolumn{2}{|c|}{148.15} & \multicolumn{2}{|c|}{249.67} \\
\hline \multirow[t]{4}{*}{0.05} & 25 & 53.98 & 53.96 & $148.46(0.02)$ & $149.80(0.04)$ & $449.82(35.15)$ & $252.00(0.14)$ \\
\hline & 50 & 54.07 & 54.07 & $150.64(0.01)$ & $150.26(0.07)$ & $254.61(0.02)$ & $253.62(0.13)$ \\
\hline & 250 & 54.06 & 54.07 & $150.37(0.00)$ & $150.39(0.04)$ & $253.87(0.00)$ & $253.92(0.02)$ \\
\hline & LR-CCSD & \multicolumn{2}{|c|}{54.62} & \multicolumn{2}{|c|}{153.19} & \multicolumn{2}{|c|}{259.40} \\
\hline \multirow[t]{4}{*}{0.10} & 25 & 57.83 & 57.57 & $163.62(0.03)$ & $165.42(0.13)$ & $462.00(22.55)$ & $282.05(0.25)$ \\
\hline & 50 & 57.99 & 57.99 & $166.46(0.02)$ & $166.11(0.05)$ & $284.81(0.03)$ & $283.96(0.22)$ \\
\hline & 250 & 57.99 & 58.00 & $166.19(0.00)$ & $166.23(0.02)$ & $284.30(0.00)$ & $284.26(0.21)$ \\
\hline & LR-CCSD & \multicolumn{2}{|c|}{58.72} & \multicolumn{2}{|c|}{170.76} & \multicolumn{2}{|c|}{294.16} \\
\hline \multirow[t]{4}{*}{0.15} & 25 & 65.85 & 64.97 & $195.14(0.07)$ & $201.06(0.17)$ & $557.18(114.72)$ & $353.66(0.57)$ \\
\hline & 50 & 66.07 & 66.06 & $202.51(0.03)$ & 202.24(0.09) & $357.02(0.05)$ & $356.37(0.20)$ \\
\hline & 250 & 66.05 & 66.08 & $202.45(0.00)$ & $202.49(0.04)$ & $357.26(0.00)$ & $357.10(0.10)$ \\
\hline & LR-CCSD & \multicolumn{2}{|c|}{67.22} & \multicolumn{2}{|c|}{212.20} & \multicolumn{2}{|c|}{381.68} \\
\hline \multirow[t]{4}{*}{0.20} & 25 & 82.03 & 79.89 & $279.06(0.35)$ & $294.06(0.89)$ & $520.61(84.68)$ & $564.50(1.38)$ \\
\hline & 50 & 82.57 & 82.54 & $296.86(0.62)$ & $295.83(1.67)$ & $564.25(16.84)$ & $566.94(0.89)$ \\
\hline & 250 & 82.56 & 82.60 & $296.71(0.55)$ & $296.44(0.06)$ & $571.44(0.71)$ & $571.63(1.73)$ \\
\hline & LR-CCSD & \multicolumn{2}{|c|}{84.83} & \multicolumn{2}{|c|}{328.71} & \multicolumn{2}{|c|}{682.10} \\
\hline
\end{tabular}


typically in error by less than $1 \%$. This is consistent with our discussion in Sec. III B where we argue that the dynamical DMRG method suffers from using the same set of DMRG basis states to represent both the zeroth-order DMRG vector as well as the response and perturbation vectors. Thus, for small $M$ there simply are not enough DMRG states to yield a meaningful result in the dynamical DMRG. Both methods converge as $M$ increases. For the most accurate calculations $(M=250)$, although both methods perform well, the dynamical DMRG polarizabilities appear slightly better than the analytic DMRG polarizabilities. However, this appears to be related to the instabilities in the convergence of the analytic DMRG response sweeps; whereas the oscillations in the dynamical DMRG sweeps vanish for larger $M$, they still remain for the analytic DMRG sweeps. From the $2 \sigma$ values, we see that currently we can only conclude that the analytic and dynamical DMRG response methods are comparable for larger $M$.

Observing the trends in the polarizabilities, we see that the polarizabilities increase as the applied frequency increases, which is what one would expect since we are approaching the first excitonic ${ }^{1} B_{u}$ pole. We are not able to converge our response calculations very close to a pole because of the large norm in $\mathbf{c}^{[1]}$. The standard solution to this is to include a small imaginary broadening in $\omega$. However, a straightforward incorporation of broadening leads to complex operators in the analytic theory which we have not yet implemented.

It is often the case that one wishes to determine an entire spectrum, i.e., some response property for a very large range of $\omega$. While in the dynamical DMRG this is usually performed by scanning through $\omega$ (with some small imaginary component) and performing a response calculation for each frequency, it may be more appropriate in the analytic response approach to adopt a different strategy. The coupledperturbed response equations may be viewed as a linear eigenvalue problem for the excitation energies (i.e., poles) and may be solved in this way, in the same way that the timedependent Hartree-Fock or time-dependent density functional equations are solved as an eigenvalue problem to obtain excitation energies. Once a sufficient number of poles are obtained, the spectrum can then be reconstructed analytically.

Comparing the DMRG polarizabilities and the CC polarizabilities, we see that the $\mathrm{CC}$ polarizabilities are generally quite good even at the singles and doubles level. (They appear to consistently overestimate the polarizability by only a few percent). This is not surprising since by virtue of the one-electron nature of the dipole operator, the linear polarizability only samples states with single-excitation character relative to the ground state. Such excited states are well captured by CCSD theory. However, earlier studies indicate that the overall spectrum in conjugated systems (including, e.g., doubly excited and triplet excited states) is poorly reproduced by CC theory, ${ }^{39}$ and so we would expect much larger discrepancies between the CC and DMRG description of third-order nonlinear optical response.

In Fig. 3 we plot the static active space and total polarizabilities $(\omega=0)$ per monomer calculated using the analytic

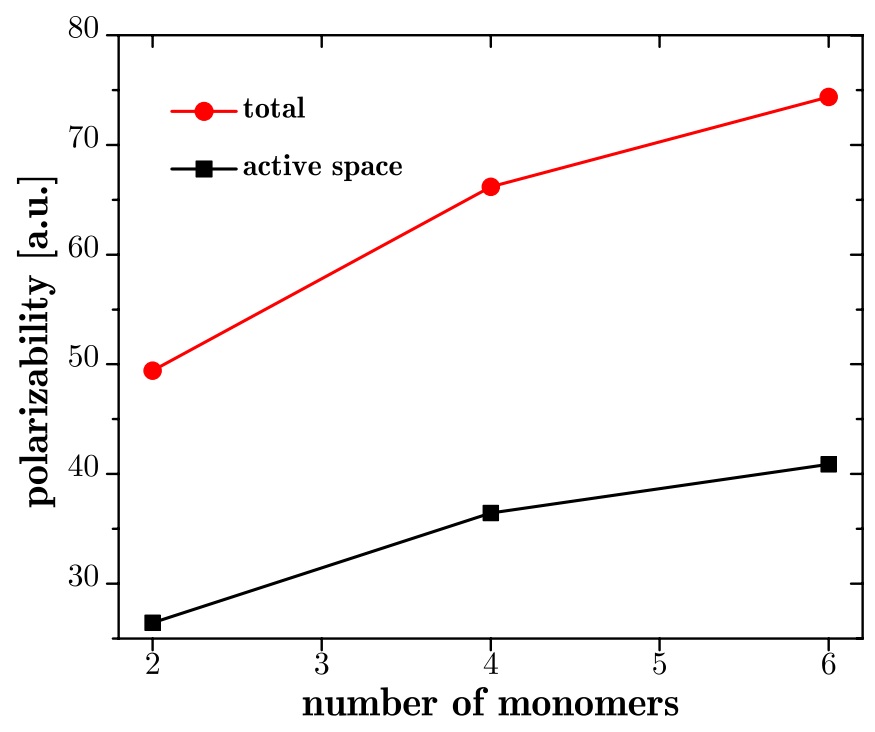

FIG. 3. (Color online) Scaling of the total and active space polarizabilities per monomer.

DMRG response theory as a function of the number of diacetylene monomers in the calculation. The total polarizability for the DMRG calculations is obtained using the core correction from the linear-response CC calculations, i.e.,

$$
\alpha_{\mathrm{DMRG}}^{\mathrm{tot}}=\alpha_{\mathrm{CC}}^{\mathrm{tot}}-\alpha_{\mathrm{CC}}^{\mathrm{act}}+\alpha_{\mathrm{DMRG}}^{\mathrm{act}} .
$$

We see a slow saturation of the polarizability per monomer as a function of the chain length, although the polarizability is not yet fully saturated at the 6-ODA level. While larger basis set calculations and calculations on longer chains are necessary to obtain a definitive conclusion, we note that our results are consistent with early semiempirical calculations which indicate an onset of saturation between 2-ODA and 3-ODA. ${ }^{40}$

\section{CONCLUSIONS}

In the current work we have described an analytic approach to the calculation of response quantities in the DMRG. The analytic response method is familiar from other electronic structure theories but has not so far been developed within the DMRG. The analytic response implementation does not change the computational cost of the groundstate DMRG calculation by more than a constant factor. Compared to the popular dynamical DMRG approach, we find that the analytic response method produces considerably more accurate response quantities when using a small number of DMRG states, without any greater computational cost. While it is simpler within the dynamical DMRG to implement higher-order response properties and complex frequencies, based on our investigations, the improved accuracy of the analytic response approach may justify the additional implementation effort. In future work, we will explore both higher-order response quantities and determination of complete spectra using the analytic DMRG response approach. 


\section{ACKNOWLEDGMENTS}

Support from the Cornell Center for Materials Research, the National Science Foundation through Grant No. CHE0645380, and the Department of Energy, Office of Science through Award No. DE-FG02-07ER46432 is acknowledged.

${ }^{1}$ S. R. White, Phys. Rev. Lett. 69, 2863 (1992).

${ }^{2}$ U. Schollwöck, Rev. Mod. Phys. 77, 259 (2005).

${ }^{3}$ S. R. White and R. L. Martin, J. Chem. Phys. 110, 4127 (1999).

${ }^{4}$ G. K.-L. Chan and M. Head-Gordon, J. Chem. Phys. 116, 4462 (2002).

${ }^{5}$ D. Ghosh, J. Hachmann, T. Yanai, and G. K.-L. Chan, J. Chem. Phys. 128, 144117 (2008)

${ }^{6}$ K. H. Marti, I. M. Ondik, G. Moritz, and M. Reiher, J. Chem. Phys. 128, 014104 (2008).

${ }^{7}$ G. K.-L. Chan, M. Kállay, and J. Gauss, J. Chem. Phys. 121, 6110 (2004).

${ }^{8}$ Y. Kurashige and T. Yanai, J. Chem. Phys. (unpublished).

${ }^{9}$ D. Zgid and M. Nooijen, J. Chem. Phys. 128, 144116 (2008).

${ }^{10}$ J. Hachmann, J. J. Dorando, M. Avilés, and G. K.-L. Chan, J. Chem. Phys. 127, 134309 (2007).

${ }^{11}$ G. Kotliar, Y. S. Savrasov, K. Haule, V. S. Oudovenko, O. Parcollet, and C. A. Marianetti, Rev. Mod. Phys. 78, 865 (2006).

${ }^{12}$ R. McWeeny and B. T. Sutcliffe, Methods of Molecular Quantum Mechanics (Academic, New York, 1969).

${ }^{13}$ C. Jamorski, M. E. Casida, and D. R. Salahub, J. Chem. Phys. 104, 5134 (1996).

${ }^{14}$ H. Koch, H. J. A. Jensen, P. Jørgensen, and T. Helgaker, J. Chem. Phys. 93, 3345 (1990).

${ }^{15}$ J. Olsen and P. Jørgensen, J. Chem. Phys. 82, 3235 (1985).

${ }^{16}$ T. Kobayashi, K. Sasagane, F. Aiga, and K. Yamaguchi, J. Chem. Phys. 110, 11720 (1999).

${ }^{17}$ J. Olsen and P. Jørgensen, in Modern Electronic Structure Theory, Advanced Series in Physical Chemistry Vol. 2, edited by D. Yarkony (World Scientific, Singapore, 1995).

${ }^{18}$ J. E. Rice and N. C. Handy, J. Chem. Phys. 94, 4959 (1991).
${ }^{19}$ K. Sasagane, F. Aiga, and R. Itoh, J. Chem. Phys. 99, 3738 (1993).

${ }^{20}$ K. A. Hallberg, Phys. Rev. B 52, R9827 (1995).

${ }^{21}$ S. S. Ramasesha, S. K. Pati, H. R. Krishnamurthy, Z. Shuai, and J. L. Brédas, Phys. Rev. B 54, 7598 (1996).

${ }^{22}$ T. D. Kühner and S. R. White, Phys. Rev. B 60, 335 (1999).

${ }^{23}$ E. Jeckelmann, Phys. Rev. B 66, 045114 (2002).

${ }^{24}$ S. R. White and I. Affleck, Phys. Rev. B 77, 134437 (2008).

${ }^{25}$ E. Jeckelmann, Prog. Theor. Phys. Suppl. 176, 143 (2008).

${ }^{26}$ S. Rommer and S. Östlund, Phys. Rev. B 55, 2164 (1997).

${ }^{27}$ D. Zgid and M. Nooijen, J. Chem. Phys. 128, 144115 (2008).

${ }^{28}$ S. R. White, Phys. Rev. B 72, 180403 (2005).

${ }^{29}$ G. K.-L. Chan, Phys. Chem. Chem. Phys. 10, 3454 (2008).

${ }^{30}$ K. Ueda, C. Jin, N. Shibata, Y. Hieida, and T. Nishino, arXiv:cond-mat./ 0612480.

${ }^{31}$ J. L. Bredas, C. Adant, P. Tackx, A. Persoons, and B. M. Pierce, Chem. Rev. (Washington, D.C.) 94, 243 (1994).

${ }^{32}$ A. D. Becke, J. Chem. Phys. 98, 5648 (1993).

${ }^{33}$ C. Lee, W. Yang, and R. Parr, Phys. Rev. B 37, 785 (1988).

${ }^{34}$ Basis sets were obtained from the Extensible Computational Chemistry Environment Basis Set Database, Version 02/25/04, as developed and distributed by the Molecular Science Computing Facility, Environmental and Molecular Sciences Laboratory, which is part of the Pacific Northwest Laboratory, P.O. Box 999, Richland, Washington 99352, USA, and funded by the U.S. Department of Energy. The Pacific Northwest Laboratory is a multiprogram laboratory operated by Battelle Memorial Institute for the U.S. Department of Energy under Contract No. DE-AC0676RLO, 1830. Contact Karen Schuchardt for further information.

${ }^{35}$ W. J. Hehre, R. F. Stewart, and J. A. Pople, J. Chem. Phys. 51, 2657 (1969).

${ }^{36}$ H. Koch and R. J. Harrison, J. Chem. Phys. 95, 7479 (1991).

${ }^{37}$ H. Sellers, Int. J. Quantum Chem. 30, 433 (1986).

${ }^{38}$ T. D. Crawford, C. D. Sherrill, E. F. Valeev, J. T. Fermann, R. A. King, M. L. Leininger, S. T. Brown, C. L. Janssen, E. T. Seidl, J. P. Kenny, and W. D. Allen, J. Comp. Chem. 28, 1610 (2007).

${ }^{39}$ J. J. Dorando, J. Hachmann, and G. K.-L. Chan, J. Chem. Phys. 127, 084109 (2007).

${ }^{40}$ B. Kirtman, Int. J. Quantum Chem. 36, 119 (1989). 